\title{
Music-to-Color Associations of Single-line Piano Melodies in Non-Synesthetes
}

\author{
Stephen E. Palmer ${ }^{1}$, Thomas A. Langlois ${ }^{1}$ and Karen B. Schloss ${ }^{2}$ \\ ${ }^{1}$ Department of Psychology, University of California, Berkeley, CA 94720-1650, USA \\ ${ }^{2}$ Department of Cognitive, Linguistic and Psychological Sciences, Brown University, \\ Providence, RI 02912, USA
}

Supplementary Materials 
Melody 1

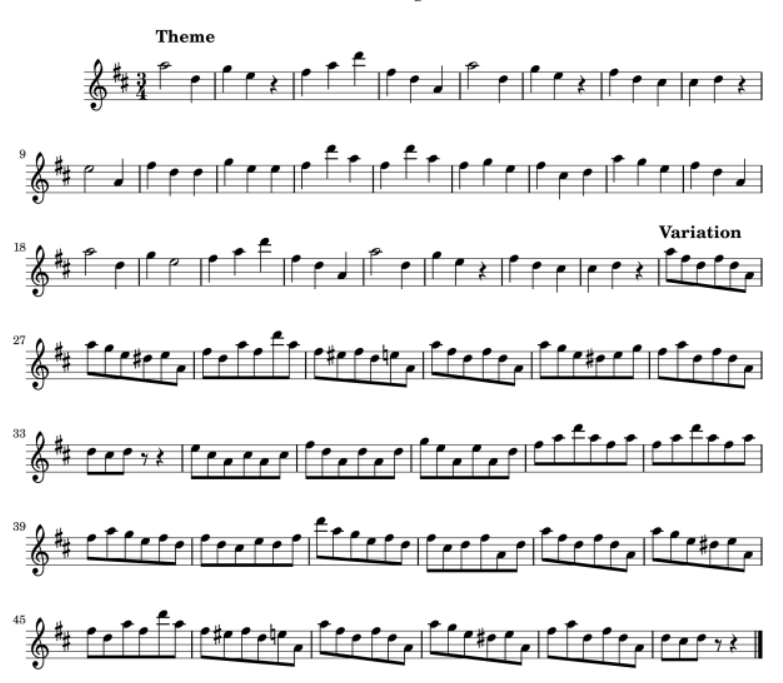

Melody 2

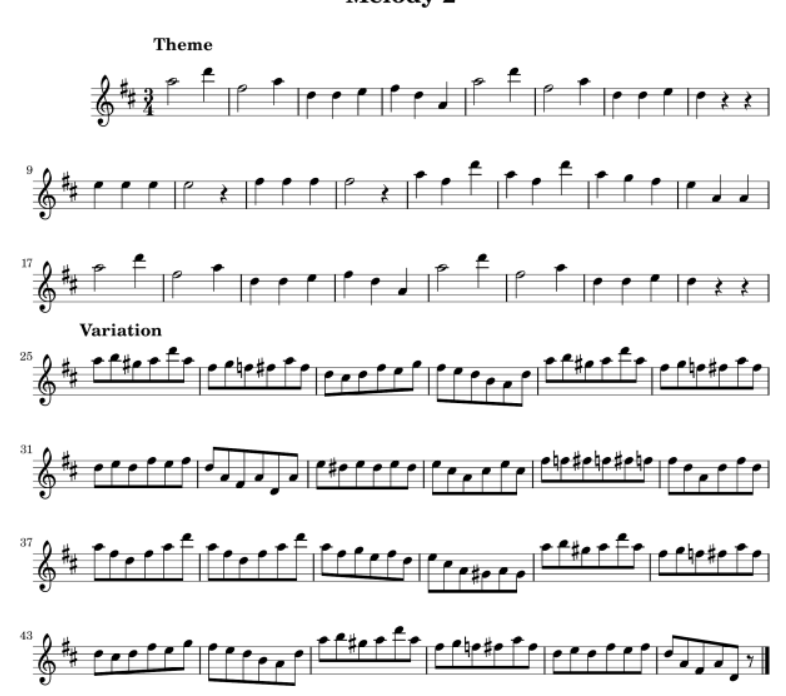

Melody 3

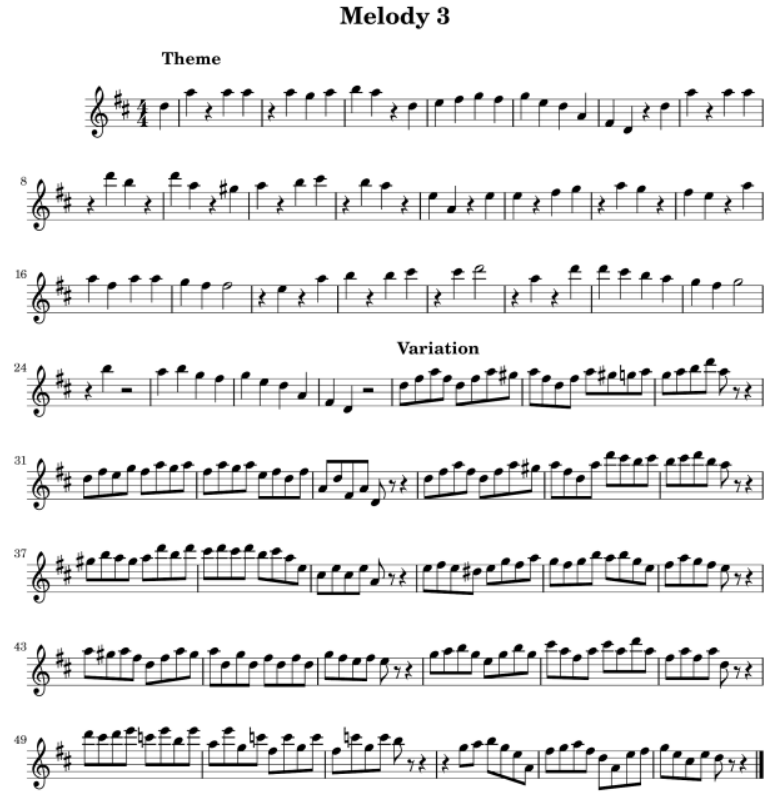

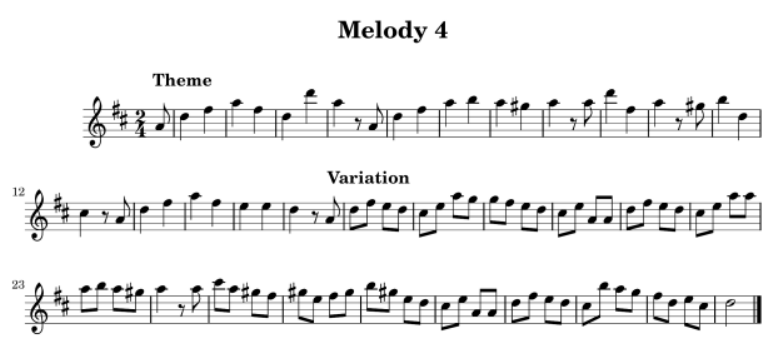

Figure S1. The four basic melodies in the major mode that were used as stimuli in the experiment. Each of the four basic melodies was based on a theme by Mozart from (1) the Duport variations in D-Major (K. 573), (2) Theme and 9 Variations in C-Major on "Lison Dormait" from Dezede's "Julie" (K. 264), (3) Theme and 6 variations in Gminor on "Helas j'ai perdu mon amant" (K. 360), and (4) Serenade No. 12 in C-minor from the 4th movement (K. 388). All themes were transposed to D-major and D-minor from their original key. The note-dense variations were based on Mozart's own variations of these themes, but were simpler and more regular than Mozart's compositions to conform to our note-rate requirements. The notes that were added to the basic melody generally produced twice as many notes/beat by interpolating a single additional note between each pair of notes in the original melody. 
Table S1. Temporal statistics of the four melodies played at their slow and fast tempi. Red entries are relevant to the determination of the modal note-value and modal note-rate for each of the four melodies.

\begin{tabular}{|c|c|c|c|c|c|c|c|c|}
\hline & \multicolumn{2}{|c|}{ MELODY 1} & \multicolumn{2}{|c|}{ MELODY 2} & \multicolumn{2}{|c|}{ MELODY 3} & \multicolumn{2}{|c|}{ MELODY 4} \\
\hline & Theme & Variation & Theme & Variation & Theme & Variation & Theme & Variation \\
\hline Time signature & $3 / 4$ & $3 / 4$ & $3 / 4$ & $3 / 4$ & $4 / 4$ & $4 / 4$ & $2 / 4$ & $2 / 4$ \\
\hline \# Measures & 25 & 25 & 24 & 24 & 27.25 & 27.25 & 16.25 & 16.25 \\
\hline \# Beats/measure & 3 & 3 & 3 & 3 & 4 & 4 & 2 & 2 \\
\hline Total \# beats & 75 & 75 & 72 & 72 & 109 & 109 & 32.5 & 32.5 \\
\hline \# Half-notes & 6 & 0 & 10 & 0 & 3 & 0 & 0 & 1 \\
\hline \# Quarter-notes & 58 & 0 & 46 & 0 & 76 & 0 & 27 & 1 \\
\hline \# Eighth-notes & 0 & 144 & 0 & 143 & 0 & 189 & 5 & 58 \\
\hline \# Half-rests & 0 & 0 & 0 & 0 & 2 & 0 & 0 & 0 \\
\hline \# Quarter-rests & 5 & 2 & 6 & 0 & 23 & 10 & 1 & 0 \\
\hline \# Eighth-rests & 0 & 2 & 0 & 1 & 0 & 9 & 4 & 1 \\
\hline Total \# notes & 69 & 148 & 62 & 144 & 104 & 208 & 37 & 61 \\
\hline$\%$ Quarter-notes & 84 & 0 & 74 & 0 & 73 & 0 & 73 & 1 \\
\hline$\%$ Eighth-notes & 0 & 97 & 0 & 99 & 0 & 91 & 13 & 95 \\
\hline Modal note-value & $1 / 4$ & $1 / 8$ & $1 / 4$ & $1 / 8$ & $1 / 4$ & $1 / 8$ & $1 / 4$ & $1 / 8$ \\
\hline \multicolumn{9}{|l|}{ SLOW TEMPO } \\
\hline Tempo (BPM) & 140 & 120 & 140 & 120 & 140 & 120 & 140 & 120 \\
\hline Total duration (sec.) & 32 & 37 & 31 & 36 & 47 & 54 & 14 & 16 \\
\hline Modal notes/min. & 140 & 240 & 140 & 240 & 140 & 240 & 140 & 240 \\
\hline Avg. notes/min. & 129 & 237 & 121 & 240 & 134 & 229 & 159 & 225 \\
\hline \multicolumn{9}{|l|}{ FAST TEMPO } \\
\hline Tempo (BPM) & 240 & 220 & 240 & 220 & 240 & 220 & 240 & 220 \\
\hline Total duration (sec.) & 19 & 20 & 18 & 20 & 27 & 30 & 8 & 9 \\
\hline Modal notes/min. & 240 & 440 & 240 & 440 & 240 & 440 & 240 & 440 \\
\hline Avg. notes/min. & 221 & 434 & 207 & 440 & 229 & 420 & 273 & 413 \\
\hline
\end{tabular}


Table S2. CIE 1931 values and Munsell values for the 32 chromatic colors and CIE 1931 values for the five achromatic colors (CIE Illuminant C). (Table from Palmer et al., 2013).

\begin{tabular}{|c|c|c|c|c|c|c|}
\hline Color & & $\mathrm{x}$ & $\mathrm{y}$ & $\mathrm{Y}$ & Hue & Value/Chroma \\
\hline \multirow[t]{4}{*}{ Red } & Saturated & 0.549 & 0.313 & 22.93 & $5 \mathrm{R}$ & $5 / 15$ \\
\hline & Light & 0.407 & 0.326 & 49.95 & $5 \mathrm{R}$ & $7 / 8$ \\
\hline & Muted & 0.441 & 0.324 & 22.93 & $5 \mathrm{R}$ & $5 / 8$ \\
\hline & Dark & 0.506 & 0.311 & 7.60 & $5 \mathrm{R}$ & $3 / 8$ \\
\hline \multirow[t]{4}{*}{ Orange } & Saturated & 0.513 & 0.412 & 49.95 & $5 \mathrm{YR}$ & $7 / 13$ \\
\hline & Light & 0.399 & 0.366 & 68.56 & $5 \mathrm{YR}$ & $8 / 6$ \\
\hline & Muted & 0.423 & 0.375 & 34.86 & $5 \mathrm{YR}$ & $6 / 6$ \\
\hline & Dark & 0.481 & 0.388 & 10.76 & $5 \mathrm{YR}$ & $3.5 / 6$ \\
\hline \multirow[t]{4}{*}{ Yellow } & Saturated & 0.446 & 0.472 & 91.25 & $5 \mathrm{Y}$ & $9 / 12$ \\
\hline & Light & 0.391 & 0.413 & 91.25 & $5 \mathrm{Y}$ & $9 / 6.5$ \\
\hline & Muted & 0.407 & 0.426 & 49.95 & $5 \mathrm{Y}$ & $7 / 6.5$ \\
\hline & Dark & 0.437 & 0.450 & 18.43 & $5 \mathrm{Y}$ & $5 / 6.5$ \\
\hline \multirow[t]{4}{*}{ Chartreuse } & Saturated & 0.387 & 0.504 & 68.56 & $5 \mathrm{GY}$ & $8 / 11$ \\
\hline & Light & 0.357 & 0.420 & 79.90 & $5 \mathrm{GY}$ & $8.5 / 6$ \\
\hline & Muted & 0.360 & 0.436 & 42.40 & $5 \mathrm{GY}$ & $6.5 / 6$ \\
\hline & Dark & 0.369 & 0.473 & 18.43 & $5 \mathrm{GY}$ & $4.5 / 6$ \\
\hline \multirow[t]{4}{*}{ Green } & Saturated & 0.254 & 0.449 & 42.40 & $3.75 \mathrm{G}$ & $6.5 / 11.5$ \\
\hline & Light & 0.288 & 0.381 & 63.90 & $3.75 \mathrm{G}$ & $7.75 / 6.25$ \\
\hline & Muted & 0.281 & 0.392 & 34.86 & $3.75 \mathrm{G}$ & $6 / 6.25$ \\
\hline & Dark & 0.261 & 0.419 & 12.34 & $3.75 \mathrm{G}$ & $3.75 / 6.25$ \\
\hline
\end{tabular}




\begin{tabular}{|c|c|c|c|c|c|c|}
\hline \multirow[t]{4}{*}{ Cyan } & Saturated & 0.226 & 0.335 & 49.95 & $5 \mathrm{BG}$ & $7 / 9$ \\
\hline & Light & 0.267 & 0.330 & 68.56 & $5 \mathrm{BG}$ & $8 / 5$ \\
\hline & Muted & 0.254 & 0.328 & 34.86 & $5 \mathrm{BG}$ & $6 / 5$ \\
\hline & Dark & 0.233 & 0.324 & 13.92 & $5 \mathrm{BG}$ & $4 / 5$ \\
\hline \multirow[t]{4}{*}{ Blue } & Saturated & 0.200 & 0.230 & 34.86 & $10 \mathrm{~B}$ & $6 / 10$ \\
\hline & Light & 0.255 & 0.278 & 59.25 & $10 \mathrm{~B}$ & $7.5 / 5.5$ \\
\hline & Muted & 0.241 & 0.265 & 28.90 & $10 \mathrm{~B}$ & $5.5 / 5.5$ \\
\hline & Dark & 0.212 & 0.236 & 10.76 & $10 \mathrm{~B}$ & $3.5 / 5.5$ \\
\hline \multirow[t]{4}{*}{ Purple } & Saturated & 0.272 & 0.156 & 18.43 & $5 \mathrm{P}$ & $4.5 / 17$ \\
\hline & Light & 0.290 & 0.242 & 49.95 & $5 \mathrm{P}$ & $7 / 9$ \\
\hline & Muted & 0.287 & 0.222 & 22.93 & $5 \mathrm{P}$ & $5 / 9$ \\
\hline & Dark & 0.280 & 0.181 & 7.60 & $5 \mathrm{P}$ & $3 / 9$ \\
\hline \multirow[t]{5}{*}{ Achromatic } & Black & 0.310 & 0.316 & 0.30 & & \\
\hline & Dark gray & 0.310 & 0.316 & 12.34 & & \\
\hline & Med Gray & 0.310 & 0.316 & 31.88 & & \\
\hline & Light Gray & 0.310 & 0.316 & 63.90 & & \\
\hline & White & 0.310 & 0.316 & 116.00 & & \\
\hline
\end{tabular}

